\title{
Measurement and simulation of diametrical and axial indirect tensile tests for weak rocks
}

\begin{abstract}
Pressuremeter has been developed considerably since its first introduction by Menard in 1955 to measure tensile strength of rocks (Silva et al., 2016) [38]. Since then, several different types of pressuremeters have been developed such as preboring pressuremeter (pBPTM), the seltboring pressuremeter (SBPMT), the cone pressuremeter either pushed (PCPMT) or driven (DCPMT) in place, and the pushed Shelby tube pressuremeter (PSPMT). However, none of these devices applicable to test the tensile strength of weak rock, hard soil or highly weathered material. The ability and reliability of new pressuremeter device called H-Ometer in obtaining the indirect tensile strength of artificial weak rocks in both axial and diametrical testing positions are investigated in this study. An established finite element model, LUSAS were also used to simulate the laboratory tests conducted using H-Ometer in order to validate its accuracy. The study showed a good agreement between experimental results and that obtained from finite element simulation. The percentage difference of the diametrical model is $11.7 \%$ which is slightly higher than axial model, 3.6\%. The differences can be considered small and acceptable for both models. The finite element results of both diametrical and axial tests showed a variation of $1.3 \%$, which is negligible and showed that the tensile strength test can be carried out on either position. However experimentally, the axial model is a preferred testing model for testing indirect tensile strength using $\mathrm{H}$-Ometer. The scatter of results indicates that the H-Ometer is a new tensile testing device that can be used with reasonably good results to test brittle geomaterials. Another advantage of this test is the portability of the equipment which can be used in both laboratory and in-situ.
\end{abstract}

Keyword : H-Ometer; Weak rocks; Tensile strength; Diametrical; Axial; Finite element 